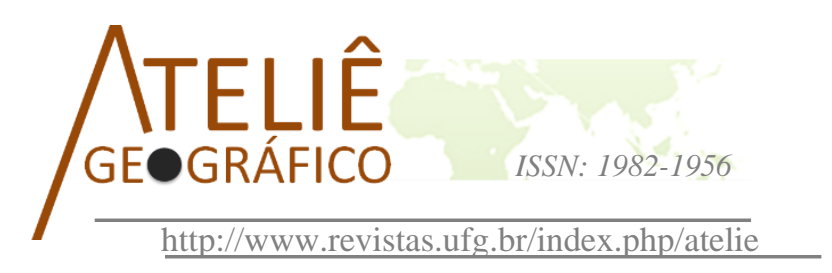

\title{
Geografia e Escalas: O lugar das escalas cartográfica, espacial e geográfica
}

\author{
Geography and Scales: The place of the concepts of \\ cartographic scale, spatial scale and geographical scale
Geografía y Escalas: El lugar de las escalas cartográfica, espacial y geográfica

\author{
Ivanilton José de Oliveira \\ Universidade Federal de Goiás (UFG) \\ oliveira@ufg.br \\ Patrícia de Araújo Romão \\ Universidade Federal de Goiás (UFG) \\ patricia_romao@ufg.br
}

\begin{abstract}
Resumo
Para demonstrar o lugar dos conceitos das escalas cartográfica, espacial e geográfica, e entender sua influência e os sentidos que lhes são atribuídos, apresentam-se contribuições oriundas de uma pesquisa bibliométrica. A metodologia consistiu em pesquisa bibliográfica exploratória de artigos e livros, em bases digitais disponíveis no Google Scholar e no Scopus-Elsevier, cuja seleção das obras privilegiou um inventário quali-quantitativo dos trabalhos publicados. Como resultado da análise, destaca-se um entendimento da escala a partir de dimensões filosóficas, políticas e sociais, além do aspecto espacial. Em relação ao uso dos conceitos nas publicações, a escala espacial é absolutamente majoritária. E quanto à reflexão realizada sobre os conceitos, sobressai a ideia de que, enquanto na Geografia há ainda certa indefinição quanto ao conceito de escala geográfica, em áreas como a Ecologia a adoção do termo "escala espacial" é pacífica, ao menos do ponto de vista metodológico. E, por fim, muitas das críticas à analogia cartográfica demonstram o equívoco de associar o mapa apenas às restrições impostas por sua escala de representação.
\end{abstract}

Palavras-chave: escala, escala espacial, escala geográfica, escala cartográfica.

\begin{abstract}
In order to demonstrate the place of the concepts of cartographic scale, spatial scale and geographic scale, and to understand their influence and the meanings they provide, contributions from a bibliometric research are presented. The methodology consisted of exploratory bibliographic research of articles and books, in digital databases
\end{abstract}


available at Google Scholar and Scopus-Elsevier, whose selection privileged a qualitative and quantitative inventory of the published works. As a result, in addition to the spatial aspect, there is an understanding of the scale in terms of philosophical, political and social dimensions. Regarding the use of the concepts in publications, the spatial scale is absolutely the majority. And as for the reflection carried out on the concepts, the idea stands out that, while in Geography there is still some uncertainty regarding the concept of geographical scale, in areas such as Ecology, the adoption of the term "spatial scale" is evident, from the methodological point of view. And, finally, many of the criticisms of the cartographic analogy demonstrate the mistake of associating the map only to the restrictions imposed by its scale.

Keywords: scale, spatial scale, geographical scale, cartographic scale.

\begin{abstract}
Resumen
Para demostrar el lugar de los conceptos de las escalas cartográfica, espacial y geográfica, y para comprender su influencia y sus significados, se presentan aportes de una investigación bibliométrica. La metodología consistió en una búsqueda bibliográfica exploratoria de artículos y libros, en bases digitales disponibles en Google Scholar y Scopus-Elsevier, cuya selección privilegió un inventario cualicuantitativo de trabajos publicados. Como resultado del análisis, existe una comprensión de la escala desde las dimensiones filosófica, política y social, además del aspecto espacial. En cuanto al uso de conceptos en las publicaciones, la escala espacial es absolutamente mayoritaria. Y en cuanto a la reflexión realizada sobre los conceptos, se destaca la idea de que, si bien en Geografía aún existe cierta incertidumbre sobre el concepto de escala geográfica, en áreas como Ecología es evidente la adopción del término "escala espacial", desde el punto de vista metodológico. Y, finalmente, muchas de las críticas a la analogía cartográfica demuestran el error de asociar el mapa solo a las restricciones impuestas por su escala. Palabras clave: escala, escala espacial, escala geográfica, escala cartográfica.
\end{abstract}

\title{
Introdução
}

A escala é um dos conceitos-chave de grande parte das pesquisas geográficas, e mesmo de outras áreas do conhecimento, por envolver um recorte analítico do problema de investigação, quase sempre em função de sua dimensão ou extensão, espacial e temporal. Mas para a Geografia a questão da escala ganha uma relevância maior, em função de sua histórica associação com os mapas, que têm na escala um pressuposto básico para a representação do espaço geográfico.

E em função dessa conexão, a escala cartográfica foi e, muitas vezes, ainda é assumida como "a escala da Geografia", o que já foi alvo de inúmeras análises críticas (RACINE, RAFFESTIN, RUFFY, 1980; CASTRO, 1995; MARQUES, GALO, 2009; entre outros). Apesar disso, é possível considerar que muitas dessas desaprovações são resultantes de uma incompreensão de que a contribuição da Cartografia para a análise geográfica não é definida apenas pelas restrições impostas pela escala do mapa (OLIVEIRA, ROMÃO, 2021).

Por sua vez, a análise geográfica envolve, quase sempre, questões associadas à Cartografia, quando esta linguagem revela e comunica conhecimentos sobre o espaço no qual ocorrem os fenômenos, não se restringindo, por isso mesmo, à escala dos mapas porventura analisados. Pois, de acordo com Castro (1995), 
todo fenômeno tem uma dimensão de ocorrência, de observação e de análise mais apropriada. A escala também é uma medida, mas não necessariamente do fenômeno, mas aquela escolhida para melhor observá-lo, dimensioná-lo e mensurá-lo.

Além das escalas cartográfica e geográfica, outro conceito permeia necessariamente essa discussão: a escala espacial. Muitas vezes tomada como sinônimo do que seria uma escala geográfica, esse vocábulo costuma ser mais empregado no sentido específico da dimensão ou extensão espacial de um fenômeno.

Por fim, cabe também aqui a referência à escala temporal, tendo em vista que todo fenômeno geográfico possui não apenas uma espacialidade, mas também uma historicidade. Logo, é possível (e, de certa forma, necessário) compreender o fenômeno com base em sua dinâmica espaço-temporal.

Partindo dessas constatações, o objetivo deste trabalho é, com base numa pesquisa do tipo bibliométrica, demonstrar o lugar de cada conceito (escala cartográfica, escala espacial e escala geográfica) em meio à produção acadêmica, para compreender seu alcance e os sentidos que lhes são atribuídos nos trabalhos de cunho geográfico (mas não necessariamente realizados apenas por geógrafos). Como a escala temporal não costuma ser motivo de controvérsias, dada sua assunção como recorte específico da temporalidade da investigação sobre um fenômeno (ou da própria manifestação deste), optou-se por não incluir este último conceito na análise proposta.

\section{Procedimentos metodológicos}

Realizou-se a identificação dos textos com base em uma pesquisa bibliográfica exploratória, a partir da revisão de publicações em formato de artigos e livros. Trata-se de uma seleção de obras com base em uma linha de raciocínio que privilegiou o inventário quali-quantitativo dos trabalhos publicados e acessíveis pelas bases de dados digitais, não sendo o intuito aqui a produção de um estado da arte sobre o assunto. No entanto, destacase que essa escolha implica em omitir certas publicações, por não constarem do rol de textos disponíveis nas bases selecionadas ou por somente existirem em versões impressas ou inacessíveis.

Dadas as facilidades hodiernas, de disponibilidade de uma vasta produção bibliográfica na forma de artigos em periódicos ou mesmo livros em meio digital, com acesso via Internet, em grande parte sem restrições de uso - mas também em função do momento em que este artigo é produzido, em meio ao isolamento social promovido pela pandemia internacional do coronavírus, que cerceou não só a mobilidade, mas inclusive o acesso direto a obras só disponíveis em bibliotecas físicas -, optou-se por realizar uma pesquisa bibliométrica exclusivamente ancorada nas bases de dados digitais de acesso livre pela Internet, ou liberadas por meio de assinaturas para instituições de ensino superior.

As fontes de acesso às obras foram escolhidas em função de sua abrangência e por sua reconhecida importância no mundo acadêmico, como referências para pesquisas. A primeira delas foi o Google Scholar (https://scholar.google.com/), ou Google 
Acadêmico, como esse buscador é nomeado na versão brasileira, reconhecido pela facilidade de acesso e amplitude do catálogo de obras.

De acordo com Noruzi (2005, p. 170), “[o] Google Acadêmico proporciona um novo método de localização de artigos potencialmente relevantes sobre determinado assunto, por meio da identificação de artigos subsequentes que citam um artigo publicado anteriormente". O autor considera que "[em] comparação a bases de dados comerciais, ele complementa as necessidades do pesquisador, fornecendo acesso a recursos não cobertos por indexadores tradicionais"1 (NORUZI, 2005, p. 180, tradução nossa).

A segunda base foi o Scopus Elsevier (https://www.scopus.com/), a maior base de dados do mundo de abstracts e de citação de literatura peer-reviewed e fontes web de qualidade (SANTOS, 2011), mas de uso restrito a assinantes.

Para padronizar as buscas dos trabalhos, definiu-se uma pesquisa somente a partir dos títulos, por se acreditar que a discussão dos trabalhos seria necessariamente centrada nos conceitos de escala, com enfoque teórico, metodológico ou mesmo aplicado. Foram utilizadas como palavras-chave os seguintes termos: "escala geográfica", "escala cartográfica", "escala espacial", "escala de análise espacial" e "escala de análise geográfica", e além do português, foram usados os termos equivalentes em espanhol, inglês e francês. Num primeiro momento, as buscas foram feitas de forma isolada, para conhecermos a quantidade de trabalhos relacionados a cada vocábulo, e na etapa seguinte, sempre conjugados, conforme as ferramentas de cada base de dados, para seleção dos trabalhados efetivamente associados a abordagens teórico-conceituais.

Dadas as similaridades linguísticas, optou-se por agrupar os resultados encontrados na pesquisa para publicações em português e espanhol, cujos vocábulos fossem idênticos. E foi necessário fazer a pesquisa em inglês para dois termos correlatos, geographic scale e geographical scale, porque ambos são utilizados nas publicações. Embora o adjetivo geographical seja mais antigo, de acordo com o Online Etymology Dictionary (OED, 2020), ele tem sido preterido contemporaneamente por sua variante mais curta, geographic. Em contrapartida, em função da construção gramatical em inglês, não foi preciso fazer a busca nesse idioma para o termo spatial scale analysis, que já estaria contido em spatial scale; bem como para os termos geographic/geographical scale analysis, contidos em geographic/geographical scale.

\section{Os conceitos na produção acadêmica}

Os resultados quantitativos das buscas realizadas nas bases de dados digitais surpreenderam pelo montante de textos com títulos contendo não os termos "escala cartográfica" ou "escala geográfica", mas sim "escala espacial" (ou spatial scale, em inglês, e échelle spatiale, em francês). E em número muito superior aos demais termos, especialmente nas buscas em inglês e francês.

\footnotetext{
1 "In comparison to commercial databases, it complements the researcher's needs by providing access to resources not covered by traditional citation indexes"
} 


\section{Google Scholar}

Os resultados no Google Scholar (Tabela 1) apresentaram algumas falhas, como a repetição de títulos (poucos), em função da existência de mais de um acesso (link), e em alguns casos também a inclusão de obras em outro idioma (apenas para português e espanhol), mesmo com as especificações na configuração e nas ferramentas de busca - o que exigiria um grande esforço para se obter a acurácia na mensuração dos dados, já que alguns termos resultaram em milhares de respostas.

Por outro lado, o Google Scholar incluiu textos de referência, como alguns capítulos de livros publicados somente no formato impresso, mas digitalizados e disponibilizados em algum site - embora nem todos autorizados para leitura na íntegra. Cabe ressaltar que não foram encontrados textos relacionados aos termos "escala de análise espacial", em português; "escala de análisis geográfica" e "escala de análisis espacial", em espanhol; e "échelle d'analiyse spatiale" e "échelle d'analiyse géographique", em francês.

Quadro 1: Pesquisa realizada no Google Scholar

\begin{tabular}{|c|c|}
\hline Palavras-chave em português e espanhol & Número de obras \\
\hline escala geográfica (POR e ESP) & 51 \\
\hline escala cartográfica (POR e ESP) & 21 \\
\hline escala espacial (POR e ESP) & 87 \\
\hline escala de análise geográfica (POR) & 2 \\
\hline Palavras-chave em inglês & Número de obras \\
\hline geographical scale & 189 \\
\hline geographic scale & 227 \\
\hline cartographic scale & 8 \\
\hline map scale & 704 \\
\hline spatial scale & 3.820 \\
\hline Palavras-chave em francês & Número de obras \\
\hline échelle géographique & 7 \\
\hline échelle cartographique & 1 \\
\hline échelle spatiale & 56 \\
\hline
\end{tabular}

Fonte: Google Scholar, em português (POR): https://scholar.google.com.br, em espanhol (ESP): https://scholar.google.es/, em inglês: https://scholar.google.com/ e em francês: https://scholar.google.fr/2

2 Elaborada pelos autores, com base em pesquisa avançada, realizada entre 20 e 21 de abril de 2020 , com as opções "Encontrar artigos com todas as palavras" e "No título do artigo"; "with all of the words" e "in the title of the article"; "tous les mots suivants" e "dans le titre de l'article". 
A quantidade de publicações em língua portuguesa ou espanhola foi consideravelmente menor, se comparada à língua inglesa, em todos os termos pesquisados (considerando-se que, em inglês, cartographic scale e map scale são equivalentes ao termo 'escala cartográfica', em português e espanhol). Contudo, as publicações em português/espanhol superaram aquelas disponíveis em língua francesa. Só é possível fazer conjecturas a respeito disso, a exemplo da dominância do inglês como língua franca e, por extensão, da comunicação científica na atualidade (VAN WEIJEN, 2012), ou da possibilidade de o próprio assunto ser considerado como algo superado na produção científica (num determinado idioma, como o francês), não suscitando mais o interesse por novas publicações.

O Google Scholar organiza a sequência dos resultados a partir de um fator de relevância que considera o índice de citações do autor, a publicação na qual a pesquisa foi divulgada, a frequência com que ela é citada em artigos e teses de outras pessoas e também se o conteúdo do estudo completo tem relação com o tema que se está buscando (CARVALHO, 2019). Por isso muitas vezes a ordem dos trabalhos não corresponde, por exemplo, ao número de citações indicadas pelo próprio buscador.

Em função da grande quantidade de resultados obtidos para alguns dos termos pesquisados, optou-se arbitrariamente pela seleção e análise tão somente dos dez primeiros listados na busca conjugada de todas as palavras-chave, desde que relacionados a uma discussão teórico-conceitual, desprezando-se as repetições porventura existentes e omitindo-se os textos de caráter mais aplicado. Os Quadros 1 e 2 apresentam os trabalhos selecionados com base em tais critérios.

Quadro 2: Sequência dos primeiros trabalhos indicados pelo Google Scholar para pesquisa sobre os termos "escala geográfica", "escala cartográfica", "escala espacial" e "escala de análise geográfica/espacial"

\begin{tabular}{|c|l|l|c|}
\hline Seq. & \multicolumn{1}{|c|}{ Título } & \multicolumn{1}{|c|}{ Autor(es) } & Ano \\
\hline $1^{\circ}$ & $\begin{array}{l}\text { Escala geográfica: da ação ao } \\
\text { império? }\end{array}$ & María Laura Silveira & 2004 \\
\hline $2^{\text {o }}$ & $\begin{array}{l}\text { A escala geográfica: noção, } \\
\text { conceito ou teoria? }\end{array}$ & $\begin{array}{l}\text { Everaldo Santos Melazzo, } \\
\text { Cloves Alexandre Castro }\end{array}$ & 2007 \\
\hline $3^{\circ}$ & $\begin{array}{l}\text { O conceito de escala geográfica nos } \\
\text { estudos de mídia regional }\end{array}$ & Sônia Aguiar & 2015 \\
\hline $4^{\circ}$ & $\begin{array}{l}\text { Escala geográfica e escala cartográfica: } \\
\text { distinção necessária }\end{array}$ & $\begin{array}{l}\text { Américo José Marques, Maria } \\
\text { de Lourdes Bueno Trindade } \\
\text { Galo }\end{array}$ & 2009 \\
\hline $5^{\circ}$ & $\begin{array}{l}\text { La escala geográfica como concepto } \\
\text { integrador en la comprensión de } \\
\text { problemas socio-ambientales }\end{array}$ & $\begin{array}{l}\text { Naxhelli Ruiz Rivera, } \\
\text { Leopoldo Galicia }\end{array}$ & 2016 \\
\hline $6^{\circ}$ & $\begin{array}{l}\text { O uso da escala geográfica na saúde } \\
\text { pública: as escalas da leishmaniose } \\
\text { visceral }\end{array}$ & $\begin{array}{l}\text { Patrícia Sayuri Silvestre } \\
\text { Matsumoto, Lourdes } \\
\text { Aparecida Zampieri } \\
\text { D'Andrea }\end{array}$ & 2019 \\
\hline
\end{tabular}




\begin{tabular}{|c|l|l|c|}
\hline Seq. & \multicolumn{1}{|c|}{ Título } & \multicolumn{1}{|c|}{ Autor(es) } & Ano \\
\hline $7^{\mathbf{0}}$ & $\begin{array}{l}\text { Escala geográfica e } \\
\text { transnacionalização: análise sobre } \\
\text { movimentos sociais e de mulheres }\end{array}$ & Dominique Masson & 2006 \\
\hline $8^{\circ}$ & $\begin{array}{l}\text { Escala geográfica, "construção social } \\
\text { da escala" e "política de escalas" }\end{array}$ & Marcelo Lopes de Souza & 2013 \\
\hline $9^{\circ}$ & $\begin{array}{l}\text { A escala cartográfica na ponta dos } \\
\text { dedos: contribuição das maquetes } \\
\text { táteis na construção da noção de } \\
\text { proporção no espaço vivido }\end{array}$ & $\begin{array}{l}\text { Flávia Gabriela Domingos } \\
\text { Silva }\end{array}$ & 2015 \\
\hline $10^{\circ}$ & $\begin{array}{l}\text { O conceito de escala geográfica e a } \\
\text { formação inicial de professores de } \\
\text { geografia }\end{array}$ & $\begin{array}{l}\text { Francisco Tomaz de Moura } \\
\text { Júnior }\end{array}$ & 2020 \\
\hline
\end{tabular}

${ }^{1}$ Capítulo do livro Os conceitos fundamentais da pesquisa sócio-espacial (SOUZA, 2013), não disponível para acesso livre na internet. Fonte: Google Scholar, em português (POR): https://scholar.google.com.br, e em espanhol (ESP): https://scholar.google.es/. ${ }^{3}$

Duas coisas se destacam inicialmente nesse levamento: a ausência de textos considerados como referências na literatura em português/espanhol, como o próprio artigo de Castro (1995) ou o livro de Lacoste (1976), e a pouca participação de textos que discutam o conceito de escala cartográfica. No primeiro caso, a inexistência dos trabalhos pode estar relacionada ao fato de não estarem acessíveis em formato digital (como é a situação dos trabalhos de Castro (1995) e Lacoste (1976), publicados apenas em formato impresso) ou de também apresentarem menor fator de relevância no Google Scholar, quando comparados às obras listadas no Quadro 1.

Já em relação à escala cartográfica, a escassez de trabalhos certamente reflete apenas a consolidação do conceito, que geralmente comparece nas discussões a título de cotejamento em relação à escala geográfica, como se vê em Silveira (2004), Melazzo e Castro (2007) e Marques e Galo (2009), ou a partir da necessidade de adaptação para outros sentidos, como o tato, conforme propõe a dissertação de Silva (2015).

Quadro 3: Sequência dos primeiros trabalhos indicados pelo Google Scholar para pesquisa sobre os termos geographical/geographic scale, cartographic scale, map scale e spatial scale.

\begin{tabular}{|c|l|l|c|}
\hline Seq. & \multicolumn{1}{|c|}{ Título } & \multicolumn{1}{|c|}{ Autor(es) } & Ano \\
\hline $1^{\circ}$ & $\begin{array}{l}\text { Scale as relation: musical metaphors of } \\
\text { geographical scale }\end{array}$ & Richard Howitt & 1998 \\
\hline $2^{\circ}$ & $\begin{array}{l}\text { Beyond state centrism: space, } \\
\text { territoriality, and geograhical scale in } \\
\text { globalization studies }\end{array}$ & Neil Brenner & 1999 \\
\hline $3^{\circ}$ & $\begin{array}{l}\text { Spatial scale problems and geostatistical } \\
\text { solutions: a review* }\end{array}$ & $\begin{array}{l}\text { Peter M. Atkinson, Nicholas } \\
\text { J. Tate }\end{array}$ & 2010 \\
\hline
\end{tabular}

\footnotetext{
${ }^{3}$ Elaborado pelos autores, com base em pesquisa avançada, realizada em 25 jul. 2020, com as opções "Encontrar artigos com todas as palavras", conectores $(O R)$ entre os termos e "No título do artigo".
} 


\begin{tabular}{|c|c|c|c|}
\hline Seq. & Título & Autor(es) & Ano \\
\hline $4^{\circ}$ & $\begin{array}{l}\text { Ecological and geographical scale: } \\
\text { parallels and potential for integration }\end{array}$ & Nathan F. Sayre & 2005 \\
\hline $5^{\circ}$ & $\begin{array}{l}\text { Hierarchies and spatial scale in process } \\
\text { geomorphology: a review }\end{array}$ & Dirk H. de Boer & 1992 \\
\hline $6^{\circ}$ & $\begin{array}{l}\text { A world in a grain of sand: towards a } \\
\text { reconceptualisation of geographical scale }\end{array}$ & Richard Howitt & 1993 \\
\hline $7^{\circ}$ & Exploring Spatial Scale in Geography* & Christopher D. Lloyd & 2014 \\
\hline $8^{\circ}$ & $\begin{array}{l}\text { Experienced and Novice Teachers' } \\
\text { Concepts of Spatial Scale* }\end{array}$ & $\begin{array}{l}\text { M. Gail Jones, Thomas } \\
\text { Tretter, Amy Taylor, Tom } \\
\text { Oppewal }\end{array}$ & 2008 \\
\hline $9^{\circ}$ & $\begin{array}{l}\text { Exploring the Effects of Geographic Scale } \\
\text { on Spatial Learning }\end{array}$ & $\begin{array}{l}\text { Jiayan Zhao, Mark Simpson, } \\
\text { Jan Oliver Wallgrün, Pejman } \\
\text { Sajjadi, Alexander Klippel }\end{array}$ & 2020 \\
\hline $10^{\circ}$ & Basic Theory of Map Scale* & $\begin{array}{l}\text { Chuanxin Zhang, Tigao Zhu, } \\
\text { Shengwu } \mathrm{Hu}\end{array}$ & 2009 \\
\hline
\end{tabular}

Importante notar que mesmo entre as mil primeiras referências não havia um único trabalho utilizando o termo cartographic scale no título - o que reforça a ideia de o conceito cartográfico de escala ser algo já alicerçado na literatura sobre o assunto.

Por fim, a busca no Google Scholar em francês resultou em apenas uma obra, relacionada ao conceito de escala geográfica: Le choix de l'échelle géographique pour un diagnostic pertinent, de Gérard-François Dumont, publicado como capítulo de um livro ${ }^{5}$ (DUMONT, 2012), e infelizmente de acesso restrito pela Internet.

\section{Scopus-Elsevier}

As pesquisas realizadas nas bases de dados do Scopus (Tabela 2) resultaram em um montante de trabalhos bem menor que aquele visto no Google Scholar. Cabe ressaltar, todavia, que o Scopus tem um grau de exigência elevado para aceitar a indexação de periódicos, com uma série de critérios técnicos e administrativos a serem atendidos pelos postulantes. Ademais, como esse rol de revistas está majoritariamente relacionado a publicações em inglês, não foi sequer necessário elaborar tabelas para os resultados das buscas em português/espanhol e francês, em sua maioria sem nenhum retorno.

${ }^{4}$ Elaborado pelos autores, com base em pesquisa avançada, realizada em 25 de jul. 2020, com as opções "with all of the words", conectores (OR) entre os termos e "in the title of the article".

${ }^{5}$ DUMONT, G.-F. Diagnostic et gouvernance des territoires: concepts, méthode, application. Paris: Armand Colin, 2012. 
Tabela 2: Pesquisa realizada no Scopus, para termos em inglês

\begin{tabular}{|l|c|}
\hline \multicolumn{1}{|c|}{ Palavras-chave em inglês } & Número de obras \\
\hline geographical scale & 178 \\
\hline geographic scale & 176 \\
\hline cartographic scale & 1 \\
\hline map scale & 91 \\
\hline spatial scale & 3.126 \\
\hline
\end{tabular}

Fonte: Scopus Elsevier (https://www.scopus.com/search/form.uri?display=basic) ${ }^{6}$

A pesquisa realizada para termos em português e espanhol também empregou os parâmetros de delimitação para busca somente por título e nos idiomas selecionados, tendo sido encontrados apenas 3 trabalhos, e somente para os vocábulos "escala geográfica". Já em francês, foi possível encontrar 1 artigo sobre "échelle géographique", 2 sobre "échelle cartographique" e 6 sobre "échelle spatiale". E de todos esses 12 trabalhos, nos três idiomas, apenas um se refere a uma discussão conceitual, sobre a escala geográfica (RIVERA; GALÍCIA, 2016).

Mesmo com a consulta nos quatro idiomas, foi possível encontrar apenas dez trabalhos na base do Scopus cujo escopo fosse teórico-conceitual (Quadro 3). As obras foram ordenadas pelo número de citações recebidas. De forma semelhante ao que ocorreu na busca realizada no Google Scholar, os textos que relatam pesquisas aplicadas empregando o conceito de spatial scale foram dominantes, e somente 2 dentre estes apresentavam alguma reflexão sobre o tema. De todo o rol de mais de 3 mil obras, os dois primeiros selecionados para o quadro (BRENNER, 1999 e 1998) apareceram na $7^{\mathrm{a}}$ e na $14^{\mathrm{a}}$ posições da lista de buscas do Scopus; e o terceiro (HOWITT, 1998), apenas na $51^{\mathrm{a}}$ colocação.

Quadro 3: Sequência dos primeiros trabalhos indicados pelo Scopus para pesquisa sobre os termos "escala geográfica", "escala cartográfica", "escala espacial”, "escala de análise geográfica/espacial", em inglês, português, espanhol e francês.

\begin{tabular}{|c|l|l|c|}
\hline Seq. & \multicolumn{1}{|c|}{ Título } & Autor(es) & Ano \\
\hline $1^{\circ}$ & $\begin{array}{l}\text { Beyond state-centrism? Space, } \\
\text { territoriality, and geographical scale } \\
\text { in globalization studies }\end{array}$ & Neil Brenner & 1999 \\
\hline $2^{\circ}$ & $\begin{array}{l}\text { Between fixity and motion: } \\
\text { accumulation, territorial organization } \\
\text { and the historical geography of } \\
\text { spatial scales }\end{array}$ & Neil Brenner & 1998 \\
\hline
\end{tabular}

\footnotetext{
${ }^{6}$ Elaborada pelos autores, com base em pesquisa avançada, realizada entre 20 e 21 de abril de 2020, com as opções TITLE ("termo procurado") AND (LIMIT-TO (LANGUAGE, "English")).
} 


\begin{tabular}{|c|c|c|c|}
\hline Seq. & Título & Autor(es) & Ano \\
\hline $3^{\circ}$ & $\begin{array}{l}\text { Scale as relation: musical metaphors } \\
\text { of geographical scale }\end{array}$ & Richard Howitt & 1998 \\
\hline $4^{\circ}$ & $\begin{array}{l}\text { Ecological and geographical } \\
\text { scale: Parallels and potential for } \\
\text { integration }\end{array}$ & Nathan F. Sayre & 2005 \\
\hline $5^{\circ}$ & $\begin{array}{l}\text { Hierarchies and spatial scale in } \\
\text { process geomorphology: a review }\end{array}$ & Dirk H. de Boer & 1992 \\
\hline $6^{\circ}$ & $\begin{array}{l}\text { A world in a grain of sand: towards a } \\
\text { reconceptualisation of geographical } \\
\text { scale* }\end{array}$ & Richard Howitt & 1993 \\
\hline $7^{\circ}$ & $\begin{array}{l}\text { Understanding spatial concepts at } \\
\text { the geographic scale without the } \\
\text { use of vision }\end{array}$ & $\begin{array}{l}\text { Robert M. Kitchin, Mark } \\
\text { Blades, Reginald G. Golledge }\end{array}$ & 1997 \\
\hline $8^{\circ}$ & $\begin{array}{l}\text { The paradox of geographical scale in } \\
\text { marx's politics* }\end{array}$ & Peter J. Taylor & 1987 \\
\hline $9^{\circ}$ & $\begin{array}{l}\text { La escala geográfica como concepto } \\
\text { integrador en la comprensión de } \\
\text { problemas socio-ambientales }\end{array}$ & $\begin{array}{l}\text { Naxhelli Ruiz Rivera, } \\
\text { Leopoldo Galicia }\end{array}$ & 2016 \\
\hline $10^{\circ}$ & $\begin{array}{l}\text { Exploring the Effects of Geographic } \\
\text { Scale on Spatial Learning }\end{array}$ & $\begin{array}{l}\text { Jiayan Zhao, Mark Simpson, } \\
\text { Jan Oliver Wallgrün, Pejman } \\
\text { Sajjadi, Alexander Klippel }\end{array}$ & 2020 \\
\hline
\end{tabular}

Pode-se observar que a lista de obras em inglês é muito semelhante à obtida com a busca no Google Scholar, mas não na mesma ordem - o que indica que o fator de relevância adotado pelo Google atenua o peso das citações, único critério utilizado no sequenciamento dos trabalhos pesquisados no Scopus. E conforme destacado anteriormente, a base de dados Scopus é majoritariamente em inglês, o que explica a ausência de textos em português e francês dentre os mais citados, com a honrosa exceção ao espanhol do trabalho de Rivera e Galicia (2016), que também aparece entre os primeiros indicados da lista do Google Scholar.

\section{As contribuições das obras selecionadas}

$\mathrm{Na}$ análise dos trabalhos obtidos com as buscas no Google Scholar e no ScopusElsevier, optou-se por uma apresentação dos textos na ordem cronológica das publicações, independentemente do idioma.

O artigo de De Boer (1992), o mais antigo da seleção realizada, apresenta uma revisão de literatura acerca das hierarquias e da escala espacial no âmbito da geomorfologia de processos. No texto, o autor assume uma concepção de escala 
dimensional, no sentido de extensão espaço-temporal do fenômeno, muito similar à proposta de Cailleux e Tricart (1956) - embora ele sequer cite quaisquer dos inúmeros trabalhos dos franceses, numa exclusão seletiva que parece comum na produção anglosaxônica, conforme bem observado por Souza (2013).

Kitchin, Blades e Golledge (1997) também se valem de uma revisão para discutir a compreensão espacial de pessoas com deficiência visual ou cegueira. Os autores apontam o fato de que grande parte dos resultados se refere a experiências conduzidas em "espaços de pequena escala" (small-scale spaces), e que, em função disso, "ainda não temos certeza de como as pessoas com deficiência visual e cegueira aprendem, armazenam e processam informações espaciais na escala geográfica"7 (KITCHIN; BLADES; GOLLEDGE, 1997, p. 225, tradução nossa).

Howitt (1998), num artigo curto mas muito didático, destaca três metáforas importantes acerca da escala: 1) como 'tamanho', ou seja, a escala cartográfica (map scale) como metáfora da escala geográfica; 2) como 'nível', ou uma metáfora piramidal para a escala geográfica, que remete à ideia de níveis de complexidade ou, mais simplesmente, níveis de hierarquia; e 3) como 'relação', em que o autor se vale de um interessante paralelo com a música, em que a mudança de escala não muda a natureza das notas (sua tonalidade), mas altera a relação entre os elementos reunidos (a totalidade musical). Assim,

em uma totalidade geográfica, muitos elementos permanecerão consistentes em uma análise geográfica que abrange diferentes escalas geográficas. O que muda nessa análise não são os elementos em si (as características de uma paisagem, os locais envolvidos em um processo de produção, os processos ecológicos que afetam uma formação social, as práticas culturais realizadas pelas pessoas), mas as relações que percebemos entre eles e as maneiras pelas quais podemos destacar elementos específicos para nossa análise. O que enfatizamos em uma escala pode não ser o que enfatizamos em outra $^{8}$ (HOWITT, 1998, p. 55, tradução nossa).

$\mathrm{O}$ autor conclui seu raciocínio informando que no mundo real da geopolítica a escala é um elemento fundamental, com políticas de escala da espacialidade de governos, corporações e organizações não governamentais - ideia que nos remete a Lacoste (1976) -, que constroem simultaneamente identidades diferentes em escalas diferentes usando precisamente os mesmos elementos. Portanto, isso deveria nos levar a repensar a relação entre pesquisa geográfica e ação social, e "enfrentar urgentemente as questões cruciais de

\footnotetext{
7 "we are still unsure as to how people with visual impairments and blindness learn, store and process spatial information at the geographic scale".

8 "in a geographical totality, many elements will remain consistent in a geographical analysis that spans across different geographical scales. What changes in such analysis is not the elements themselves (the features on a landscape, the sites involved in a production process, the ecological processes affecting a social formation, the cultural practices performed by people), but the relationships that we perceive between them and the ways in which we might emphasize specific elements for analytical attention. What we emphasize at one scale may not be what we emphasize at another".
} 
como agir em múltiplas escalas simultaneamente; como pensar globalmente e agir localmente, ao mesmo tempo que pensar localmente e agir globalmente (e em outras escalas, simultaneamente)" ${ }^{\prime 9}$ (HOWITT, 1998, p. 56, tradução nossa).

Os artigos de Brenner $(1998,1999)$ são uma bela exceção à francofobia dos anglo-saxões. Logo na introdução do texto de 1998, o autor recorre à obra de Lefebvre para dar o devido destaque à importância da escala. Ele também reconhece o incremento das pesquisas sobre a escala geográfica nos anos 1990, versando sobre a unidade espaçotemporal apropriada e o nível de abstração para a pesquisa histórico-empírica; processos de 'reescalonamento' como dimensões centrais da reestruturação capitalista mundial; a importância para estratégias de transformação social e política; o papel nas lutas discursivo-ideológicas pelo controle hegemônico do espaço social e político; e sobre a construção política da escala, ou seja, as escalas nas quais os processos políticos são organizados e as lutas concomitantes de atores sociais, movimentos e instituições para influenciar a sua estrutura locacional, extensão territorial e organização qualitativa.

Brenner (1998) argumenta que as estruturas escalares tanto das cidades quanto dos estados territoriais têm sido moldadas cada vez mais diretamente pela contradição entre fixidez e movimento na circulação do capital. Muito apoiado na produção de Lefebvre e de David Harvey, o autor conclui que a globalização atual pode ser interpretada como um processo multidimensional de reescalonamento, no qual cidades e estados estão sendo reterritorializados na busca conflituosa por ajustes escalares que ele chama de "glocais".

Já no texto de 1999, Brenner retoma a ideia de que o ciclo contemporâneo de globalização apresenta uma reconfiguração conflituosa do espaço social, que se desdobra simultaneamente em múltiplas escalas geográficas sobrepostas. Para o autor, esse processo descentrou a escala nacional das relações sociais e intensificou a importância das escalas sub e supranacionais de organização territorial. Assim, o desafio metodológico resultante é "conceber configurações de escalas geográficas ao mesmo tempo como o 'andaime' territorial dentro do qual a dialética da desterritorialização e da reterritorialização se desdobra, e como o meio historicamente produzido e em constante mudança dessa dialética"10 (BRENNER, 1999, p. 69, tradução nossa).

O trabalho melhor posicionado no ranking das buscas do Google Scholar em português, de Silveira (2004) se baseia em Grataloup (1979), Racine, Raffestin e Ruffi (1980) e Castro (1995), para também ressaltar a "inconveniência da analogia escala cartográfica-escala geográfica", por reafirmar a existência de "uma concepção geométrica do espaço", mesmo reconhecendo a necessidade da escala cartográfica, como "fundamento técnico e metodológico" (SILVEIRA, 2004, p. 88).

\footnotetext{
9 "urgently tackle the crucial questions of how to act at multiple scales simultaneously; how to think globally and act locally, at the same time as thinking locally and acting globally (and at other scales simultaneously)" 10 "to conceive configurations of geographical scales at once as the territorial scaffolding within which the dialectic of de- and reterritorialization unfolds and as the historically produced, incessantly changing medium of that dialectic"
} 
Para Melazzo e Castro (2007, p. 136), por sua vez, a escala cartográfica consiste em uma "medida matemática" que é "capaz de um duplo propósito": estabelecer "a proporção entre as coisas" por um lado e uma relação "entre um mapa e a medida real no local", por outro, e cuja noção equivaleria a "uma estratégia de reprodução de uma realidade anterior e já dada".

O termo "realidade dada" é sugerido como "a noção de que a realidade seria também imutável" (MELAZZO; CASTRO, 2007, p. 136). Neste caso, a noção de "imutável" deixa transparecer a incompreensão de que os mapas, ainda que apresentem um recorte temporal definido, sejam capazes de revelar o conhecimento da dinâmica espaço-temporal dos fenômenos representados.

Por outro lado, a utilização da palavra "reprodução" por Melazzo e Castro (2007, p. 136) dá a ideia de que a realidade pode ser duplicada no mapa e de que a escala é tomada como referência única - quando, na verdade, a cartografia não almeja tal duplicação, pois necessariamente estabelece recortes e escolhas, ocultando ou ressaltando determinados aspectos do fenômeno, durante a construção da representação.

Já para Marques e Galo (2009, p. 48), "não há leitura em um mapa sem determinação da escala, assim como não há análise de fenômenos sem que seja esclarecida a escala geográfica adotada". Ao tratarem de estudos realizados na Ecologia de Paisagens, ressaltam o fato de que "é necessário considerar a área de ocorrência de determinado fenômeno", deixando clara uma concepção de que a escala geográfica se relaciona à extensão dessa área.

Em função disso, Marques e Galo (2009, p. 49) assumem a ideia de que há uma relação inversamente proporcional entre as escalas cartográfica e geográfica, pois "quanto maior for a área compreendida por um fenômeno, menor deverá ser a escala cartográfica adequada para a sua representação", e vice-versa. Contudo, os autores abordam a escala temporal como um conceito dissociado do conceito de escala geográfica, a despeito de discutirem níveis de hierarquia, comparando a dimensão espacial com o tempo de ocorrência associado aos fenômenos.

O texto seguinte, de Sayre (2005), se propõe a estabelecer uma importante ponte entre as discussões de escala na Ecologia e na Geografia Humana. Ao discutir as características ontológicas e epistemológicas da escala, o autor afirma que, em um sentido mais técnico, a escala é um atributo de como alguém observa algo, e não da coisa observada.

Ao argumentar a favor de um diálogo entre a Ecologia e a Geografia Humana em relação à definição de escala e nível, o autor afirma que

a base epistemológica para distinguir entre os níveis biológicos são os processos que podem ser identificados e estudados: o crescimento de uma planta, por exemplo, ou a competição entre plantas vizinhas por recursos limitados. Da mesma forma, os geógrafos delimitam níveis de organização social em termos de processos como 
circulação de capital, governança, reprodução social, consumo e assim por diante ${ }^{11}$ (SAYRE, 2005, p. 286, tradução nossa).

Sayre (2005) também critica o fato de que em grande parte da literatura sobre o assunto, os termos 'escala' e 'nível' são usados indistintamente (como, aliás, foi feito por Souza (2013)), e se detém sobre publicações de Sallie Marston, Neil Smith e Neil Brenner, entre outros, para demonstrar esses equívocos conceituais em discussões sobre políticas de escala, escala urbana, reescalonamento, entre outros assuntos.

Por fim, o autor reconhece não se pode postular relações hierárquicas entre os níveis, especialmente para os processos sociais, porque as instituições políticas e econômicas podem ser criadas, modificadas ou suspensas, por exemplo, de maneiras que os processos biológicos geralmente não podem (SAYRE, 2005, p. 286).

Já o artigo de Masson (2006) se propõe a "explorar o potencial de novas contribuições da geografia política e humana que coloquem a questão da escala em primeiro plano, como uma dimensão espacial central dos processos pelos quais se realiza a transnacionalização da ação coletiva" (MASSON, 2006, p. 445, grifo no original). Para tanto, a autora vislumbra a escala como uma propriedade espacial das relações sociais: a escala seria a difusão da abrangência por diferentes (e variáveis) extensões do espaço, dos diversos processos econômicos e políticos que organizam as relações sociais e a vida social.

Souza (2013), de igual modo a outras obras da literatura em português, adota o discurso crítico em relação à escala cartográfica, ressaltando a necessidade de "se emancipar o raciocínio escalar para além dos estreitos limites da cartografia [...] na pesquisa sócioespacial" (SOUZA, 2013, p. 183). Sobre a escala geográfica, o autor propõe que ela deve ser subdividida em escala do fenômeno (a abrangência física ou dos processos relacionados ao fenômeno), escala de análise (o nível analítico de apreensão do fenômeno) e escala de ação (a reflexão sobre o alcance espacial das práticas de agentes sociais).

Além de também recuperar a contribuição de Lacoste e outros autores discutidos no texto de Castro (1995), o autor dá destaque à dissertação de Bahiana (1986), além apresentar uma ampla produção de origem anglo-saxônica sobre o tema 'escala'. E apesar de inicialmente considerar um equívoco "a identificação de um número fixo e constante de níveis" para a escala "no âmbito sócio-espacial" (SOUZA, 2013, pp. 187-188), ao final o autor se rende à ideia de propor uma tipologia, ainda que não vista como "um quadro rígido de referências". A tipologia, que ele especifica como "sujeita a vários aperfeiçoamentos" (p. 199), adota uma indistinção entre 'escala' ou 'nível', e se inicia pela "escala (ou nível)" do corpo, passando pelos nanoterritórios, o local (subdividido em micro, meso e macrolocal), o regional, o nacional e, finalmente, a "escala (ou nível)" internacional (também subdividida em "de grupos de países" e "global").

\footnotetext{
11 "the epistemological basis for distinguishing among biological levels is processes that can be identified and studied: the growth of a plant, for example, or competition among neighboring plants for limited resources. In the same way, geographers delimit levels of social organization in terms of processes such as capital circulation, governance, social reproduction, consumption and so forth".
} 
O texto de Aguiar (2015) conjuga principalmente as reflexões de Castro (1995) e Souza (2013), culminando na ideia de que tipologias de escalas servem tão somente para orientar as escolhas dos níveis de análise. A autora assume a escala como "um instrumento metodológico de mão dupla, que pode partir dos desdobramentos do lugar (cidade, aldeia, bairro, quarteirão, rua etc.) ou da totalidade global (mundo, nação, região, lugar)" (AGUIAR, 2015, p. 6).

A dissertação de mestrado de Silva (2015) foi o único trabalho elencado nas buscas a tratar do conceito de escala cartográfica. Uma das ideias apresentadas pela autora é a de que a dificuldade atribuída à compreensão da escala cartográfica pelos educandos decorre, principalmente, de sua restrição à noção de proporção quantificada. Contudo, como ela destaca, a compreensão da proporcionalidade é construída, primeiramente, em termos não quantificáveis, com base em relações de primeira ordem (comparações entre as partes e, depois, das partes com o todo) e de segunda ordem (comparações das relações de primeira ordem entre si).

Essa construção incluiria, além disso, o estabelecimento de medidas perceptivas, a exemplo das comparações entre objetos por suas formas, pelo paralelismo, a igualdade de ângulos e, por fim, a perspectiva dimensional. A partir desses pressupostos, Silva (2015) conclui que a compreensão do conceito de escala cartográfica pelo aluno com deficiência visual pode, então, ser efetivada a partir do trabalho com a noção de proporção não quantificável existente no espaço de vivência desse aluno.

O texto de Rivera e Galicia (2016) também traz uma contribuição importante quanto ao acesso à literatura anglo-saxônica sobre as discussões acerca da escala. Eles iniciam o trabalho indicando que a escala "é definida mais como um atributo da observação científica do objeto, que como uma dimensão intrínseca ao objeto observado"12 (RIVERA; GALICIA, 2016, p. 138, tradução nossa). Apoiados principalmente nos trabalhos de Gibson, Ostrom e Ahn (2000) e McMaster e Sheppard (2004), os autores sintetizam que

a escala representa um conjunto de relações espaciais entre elementos organizados em diferentes tipos de relações hierárquicas, as quais são observadas por meio de uma extensão e uma resolução específicas. A escala é o instrumento epistemológico para identificar, observar e interpretar as propriedades emergentes dos fenômenos a serem estudados ${ }^{13}$ (RIVERA; GALICIA, 2016, p. 142, tradução nossa).

Matsumoto e D'Andrea (2019) fazem uma importante revisão de literatura sobre o uso do conceito de escala, em que constatam que "é ainda incipiente a discussão da

\footnotetext{
12 "la escala es definida como un atributo de la observación científica del objeto, más que como una dimensión intrínseca al objeto observado".

13 "la escala representa un conjunto de relaciones espaciales entre elementos organizados en diferentes tipos de relaciones jerárquicas, los cuales son observados a través de una extensión y uma resolución específicas. La escala es el instrumento epistemológico para identificar, observar e interpretar las propiedades emergentes de los fenómenos a estudiar".
} 
escala geográfica na saúde em uma concepção que extrapole a noção material" (MATSMUTO; D'ANDREA, 2019, p. 3826)". A parte do texto que discute propriamente a escala é apoiada em autores já anteriormente indicados, como Racine; Raffestin; Ruffy (1983), Bahiana (1986), Castro (1995), Melazzo e Castro (2007) e Souza (2013), além de Smith (2000). As autoras acreditam numa indissociabilidade entre as escalas cartográfica e geográfica, afirmando que "a escala geográfica traz subjetividade e outras acepções além da materialidade, mas sempre terá uma representação material, que se traduz na escala cartográfica" (MATSUMOTO; D’ANDREA, 2019, p. 3827)

Último dos dez trabalhados obtidos com a busca em português ou espanhol, a dissertação de mestrado de Moura Júnior (2020) também se vale da contraposição com a escala cartográfica para iniciar sua discussão, subsidiada pelos textos de Castro (1995), Marques e Galo (2009), entre outros, e culmina na afirmação de que "enquanto a escala cartográfica é uma representação estanque, momentânea e pontual, a escala geográfica é historicamente produzida, transformando-se ao longo do tempo, sendo processual e contraditória" (MOURA JÚNIOR, 2020, p. 51).

$\mathrm{Na}$ análise do conceito de escala geográfica, Moura Júnior (2020) se apoia nas reflexões de Neil Smith, sem dúvida uma das grandes referências na produção teórica sobre a escala enquanto produto social e político. Ele se vale do artigo de Vieira (2012) para elencar o que seriam os pontos centrais da teoria de Smith:

1) a escala geográfica é produzida socialmente; 2) os fenômenos socioespaciais são sempre multiescalares; 3) as escalas geográficas são diferentes em si e estão ordenadas hierarquicamente, ainda que essa hierarquia não seja totalmente rígida; 4) as diferenças proporcionam diferentes significados às escalas: local, regional, nacional e global; 5) os significados diferentes podem ser coincidentes, mas nunca idênticos; 6) o fator direcionador da produção da escala é a relação capital/trabalho sem, contudo, desconsiderar a dimensão sociocultural das relações sociais; 7) a produção da escala, a partir da relação capital/trabalho, é contraditória e não-linear (MOURA JÚNIOR, 2020, p. 53).

Por fim, o último trabalho analisado dentre aqueles resultantes das buscas no Google Scholar e no Scopus-Elsevier, de Zhao et al. (2020), também se refere a uma discussão do conceito de escala geográfica no campo do ensino-aprendizagem. Contudo, embora os autores citem uma obra que apresenta definição mais refinada acerca da escala geográfica (SAYRE, 2005), a concepção adotada no trabalho é, na verdade, a de uma escala espacial, ou seja, relativa à "extensão espacial de um fenômeno ou estudo"14 (ZHAO et al., 2020, p. 2, tradução nossa).

Com base na assunção de escala como "extensão espacial visualmente acessível a partir de um único ponto de vista", os autores conduziram experimentos com pessoas de alta e baixa habilidade espacial, para comparar como a acessibilidade visual de um

\footnotetext{
14 "which refers to the spatial extent of a phenomenon or a study".
} 
ambiente afeta a aprendizagem espacial. E concluíram que uma escala "ampliada" do ambiente (visto de um ponto mais elevado) pode ajudar na aprendizagem espacial, especialmente para participantes com baixa capacidade espacial.

\section{Considerações Finais}

A julgar pela análise das obras aqui discutidas, enquanto na Geografia existe certa indefinição quanto ao entendimento do que seja, de fato, uma escala geográfica, em áreas como a Ecologia, ao que parece, o problema teórico-conceitual acerca da escala já é algo resolvido, com a adoção do termo "escala espacial", cujo sentido é o da dimensão, da extensão do fenômeno analisado - ainda que a chamada "escala ecológica" também não se reduza apenas ao aspecto dimensional, como bem demonstrado por Sayre (2005).

A análise das obras selecionadas com base na pesquisa bibliométrica nos apresentou um grande número de outras referências, com discussões sobre a escala no âmbito social e político, como Bahiana (1986), Smith (1992, 2002), Delaney e Leitner (1997), Marston (2000), Marston et al. (2005) e Moore (2008); ou que se aprofundam na relação entre escala, espaço e tempo, a exemplo de Meentemeyer (1989), Fresco e Kroonemberg (1992) e Grataloup (2003); ou sob muitos outros aspectos, como o texto de Gibson, Ostrom e Ahn (2000) e a coletânea organizada por Sheppard e McMaster (2004). Mas infelizmente não é possível realizar a discussão de tais textos, dados os objetivos aqui propostos.

Em outro artigo (OLIVEIRA; ROMÃO, 2021) nos propusemos a também contribuir, esquematizando as relações entre as escalas cartográfica e geográfica, que perpassam, necessariamente, também pela escala espacial, deixando "claro que o constructo 'escala' contém a chamada 'escala geográfica', assim como esta integra as escalas espacial e cartográfica”, e que:

[...] as escalas espacial e cartográfica não se combinam para formar a escala geográfica, pois elas respondem tão somente ao aspecto dimensional, em função da extensão do fenômeno ou de sua representação, respectivamente. E conforme já bem demonstrado na produção bibliográfica no campo da Cartografia, os mapas são representações que contemplam apenas uma parte da realidade: aquela que interessa ao cartógrafo-geógrafo, e da qual a escala (do mapeamento) indica apenas um dos recortes. Por isso mesmo, a escala cartográfica não se justapõe totalmente à escala espacial - e menos ainda à escala geográfica.

Os trabalhos em destaque nas bases de dados demonstram, outrossim, o interesse pela compreensão da escala do ponto de vista ontológico. Neste sentido, a adjetivação da escala se torna ainda mais necessária quando se trata de compreender qual o alcance da referência utilizada. Cabe destacar, também, a diferença no enfoque dos textos em língua inglesa, em que a contraposição entre escala geográfica e escala cartográfica praticamente não aparece.

Por fim, mas não menos importante, vale ressaltar a importância de não se restringir o aspecto representacional na análise geográfica apenas às limitações impostas 
pela escala nos mapas. A linguagem cartográfica, baseada na análise e tratamento das informações, apresenta uma enorme capacidade analítica ou de síntese, com vistas a construir imagens que se prestem a revelar a geografia dos fenômenos. A escala cartográfica, portanto, influencia o nível de representação, mas não determina o alcance da informação presente em um mapa.

\section{Referências}

AGUIAR, S. O conceito de escala geográfica nos estudos de mídia regional. In: XXXVIII Congresso Brasileiro de Ciências da Comunicação. Anais (on-line). Rio de Janeiro: Intercom, 2015. Disponível em: <http://www.geografias.net.br/papers/2015/R10-3462-1.pdf>. Acesso em: 25 jul. 2020.

ATKINSON, P. M.; TATE, N. J. Spatial scale problems and geostatistical solutions: a review. The Professional Geographer, v. 52, n. 4, p. 607-623, 2010.

BAHIANA, L. C. C. Contribuição ao estudo da questão da escala na Geografia: escalas em Geografia Urbana. Dissertação (Mestrado em Geografia) - Programa de Pós-Graduação em Geografia, Universidade Federal do Rio de Janeiro, 1986.

BRENNER, N. Between fixity and motion: accumulation, territorial organization and the historical geography of spatial scales. Environment and Planning D: Society and Space, v. 16, p. 459-481, 1998. Disponível em:

$\langle$ http://citeseerx.ist.psu.edu/viewdoc/download?doi=10.1.1.469.2833\&rep=rep1\&type=pdf $>$. Acesso: em 25 jul. 2020.

BRENNER, N. Beyond state-centrism? Space, territoriality, and geographical scale in globalization studies. Theory and Society, v. 28, p. 39-78, 1999. Disponível em: < https://www.researchgate.net/publication/226180064_Beyond_State-

centrism_Space_Territoriality_and_Geographical_Scale_in_Globalization_Studies $>$. Acesso em: 25 jul. 2020.

CAILLEUX, A.; TRICART, J. Le problème de la classification des faits géomorphologiques. Annales de Géographie, v. 65, n. 349, p. 162-186, 1956. DOI: 10.3406/geo.1956.14285.

CARVALHO, L. Como usar o Google Acadêmico para pesquisar artigos científicos. In: Olhar Digital, 2019. Disponível em:

<https://olhardigital.com.br/dicas_e_tutoriais/noticia/como-usar-o-google-academico-parapesquisar-artigos-cientificos/81648>. Acesso em: 23 jul. 2020.

CASTRO, I. E. O problema da escala. In: CASTRO, I. E.; GOMES, P. C. C.; CORRÊA, R. L. (org.). Geografia: Conceitos e temas. Rio de Janeiro: Bertrand Brasil, 1995.

DE BOER, D. H. Hierarchies and spatial scale in process geomorphology: a review. Geomorphology, n. 4, p. 303-318, 1992. Disponível em: <https://www.sciencedirect.com/science/article/pii/0169555X9290026K >. Acesso em: 23 jul. 2020. 
DELANEY, D.; LEITNER, H. The political construction of scale. Political Geography, v. 16, n. 2, p. 93-97, 1997.

ÉCHELLE. In: La Langue Française. 2020. Disponível em:

https://www.lalanguefrancaise.com/dictionnaire/definition-echelle/. Acesso em: 21 abr. 2020.

FRESCO, L. O.; KROONENBERG, S. B. Time and spatial scales in ecological sustainability. Land Use Policy, v. 9, n. 3, p. 155-168, jul. 1992.

GEOGRAPHICAL. In: Online Etymology Dictionary. Disponível em:

<https://www.etymonline.com/search?q=geographical>. Acesso em: 25 jul. 2020.

GIBSON, C. C.; OSTROM, E.; AHN, T. K. The concept of scale and the human dimensions of global change: a survey. Ecological Economics, v. 32, p. 217-239, 2000. Disponível em: <https://www.sciencedirect.com/science/article/pii/S0921800999000920 >. Acesso em: 21 jul. 2020.

GRATALOUP, C. Démarches des échelles. Espaces Temps. Cachan: 10-11, p. 72-79, 1979. DOI: https://doi.org/10.3406/espat. 1979. 3032

GRATALOUP, C. Os períodos no espaço. Trad. Teresa Leon. GEOgrafia, ano 8, n. 16, p. 31-40, 2006.

HOUAISS, A.; VILLAR, M. S. Dicionário Houaiss da Língua Portuguesa. Rio de Janeiro: Objetiva, 2001.

HOWITT, R. A world in a grain of sand: towards a reconceptualisation of geographical scale. Australian Geographer, v. 24, n. 1, p. 33-44, 1993.

HOWITT, R. Scale as relation: musical metaphors of geographical scale. Area, v. 30, n. 1, p. 49-58, 1998. Disponível em: <https://rgs-

ibg.onlinelibrary.wiley.com/doi/epdf/10.1111/j.1475-4762.1998.tb00047.x >. Acesso em: 25 jul. 2020.

JONES, M. G.; TRETTER, T.; TAYLOR, A.; OPPEWAL, T. Experienced and Novice Teachers' Concepts of Spatial Scale. International Journal of Science Education, v. 30, n. 3, p. 409-429, 2008.

KITCHIN, R. M.; BLADES, M.; GOLLEDGE, R. G. Understanding spatial concepts at the geographic scale without the use of vision. Progress in Human Geography, v. 21, n. 2, p. 225- 242, 1997. Disponível em:

<https://www.researchgate.net/publication/225303255_Understanding_spatial_concepts_at_t he_geographic_scale_without_the_use_of_vision>. Acesso em: 25 jul. 2020.

LACOSTE, Y. La géographie, ça sert, d'abord, à faire la guerre. Paris: Maspero, 1976.

LLOYD, C. D. Exploring Spatial Scale in Geography. Chichester: Wiley-Blackwell, 2014. $272 \mathrm{p}$.

MARQUES, A. J.; GALO, M. L. B. T. Escala geográfica e escala cartográfica: distinção necessária. Boletim de Geografia, Maringá, v. 26/27, n. 1, p. 47-55, 2008/2009. 
MARSTON, S. A. The social construction of scale. Progress in Human Geography, v. 24, n. 2, p. 219-242, 2000. Disponível em: 〈http://www.u.arizona.edu/ compitel/marston.pdf>. Acesso em: 25 jul. 2020.

MARSTON, S. A.; JONES III, J. P.; WOODWARD, K. Human geography without scale. Transactions of the Institute of British Geographers, v. 30, p. 416-432, 2005.

MASSON, D. Escala geográfica e transnacionalização: análise sobre movimentos sociais e de mulheres. Caderno CRH, v. 19, n. 48, p. 445-459, 2006. Disponível em: <https://portalseer.ufba.br/index.php/crh/article/view/18866/12235>. Acesso em: 25 jul. 2020 .

MATSUMOTO, P. S. S.; D’ANDREA, L. A. Z. O uso da escala geográfica na saúde pública: as escalas da leishmaniose visceral. Ciências e Saúde Coletiva, v. 24, n. 10, p. 3.8253.836, 2019. DOI: 10.1590/1413-812320182410.25452017.

McMASTER, R. B.; SHEPPARD, E. Introduction: Scale and Geographic Inquiry. In: SHEPPARD, E.; MCMASTER, R. B. (ed.). Scale and geographic inquiry: nature, society, and method. Oxford: Blackwell Publishing Ltd, 2004. p. 1-22.

MEENTEMEYER, V. Geographical perspectives of space, time, and scale. Landscape Ecology, v. 3 n. 3/4, p. 163-173, 1989. Disponível em:

<http://wiki.dpi.inpe.br/lib/exe/fetch.php?media=ser457-

cst310:meentemeyer_spacetime_scale_landscapeecology_1989.pdf $>$. Acesso em: 25 jul. 2020 .

MELAZZO, E. S.; CASTRO, C. A. A escala geográfica: noção, conceito ou teoria? Terra Livre, ano 23, v. 2, n. 29, p. 133-142, 2007.

MOORE, A. Rethinking scale as a geographical category: from analysis to practice. Progress in Human Geography, v. 32, n. 2, p. 203-225, 2008. DOI: 10.1177/0309132507087647

MOURA JÚNIOR, F. T. O conceito de escala geográfica e a formação inicial de professores de geografia. 2020. Dissertação (Mestrado em Geografia) - Universidade Federal de Goiás, Jataí, 2020. 102 f.

NORUZI, A. Google Scholar: The New Generation of Citation Indexes. Libri, v. 55, p. 170180, 2005. Disponível em:

<https://www.researchgate.net/publication/28804326_Google_Scholar_The_New_Generatio n_of_Citation_Indexes>. Acesso em 23 jul. 2020.

OLIVEIRA, I. J.; ROMÃO, P. A. As escalas da geografia: pontes entre os conceitos de escala cartográfica e escala geográfica. Boletim Goiano de Geografia, v. 41: e65735, 2021. DOI: $10.5216 /$ bgg.v41.65735.

RACINE, J. B.; RAFFESTIN, C.; RUFFY, V. Echelle et action, contributions à une interprétation du mécanisme de l'échelle dans la pratique de la géographie. Geographica Helvetica, v. 35, n. 5, p. 87-94, 1980. Disponível em: <https://archiveouverte.unige.ch/unige:4319>. Acesso em: 21 abr. 2020. 
RACINE, J. B.; RAFFESTIN, C.; RUFFY, V. Escala e ação, contribuições para uma interpretação do mecanismo de escala na prática da Geografia. Revista Brasileira de Geografia. n. 45, v. 1. jan/mar. Rio de Janeiro: Fundação Instituto Brasileiro de Geografia e Estatística, 1983.

RIVERA, N. R.; GALICIA, L. La escala geográfica como concepto integrador en la comprensión de problemas socio-ambientales. Investigaciones Geográficas, n. 89, p. 137153, 2016, DOI: https://doi.org/10.14350/rig.47515.

SANTOS, G. C. Fontes de indexação para periódicos científicos: um guia para bibliotecários e editores. Campinas, SP: E-Color, 2011. (Manuais técnicos BFE; n. 6).

SAYRE, N. F. Ecological and geographical scale: parallels and potential for integration. Progress in Human Geography, v. 29, n. 3, p. 276-290, 2005. Disponível em: <https://jornada.nmsu.edu/files/bibliography/05-026.pdf〉. Acesso em: 21 abr. 2020.

SCALE. In: Merriam-Webster.com Dictionary. 2020. Disponível em: <https://www.merriam-webster.com/dictionary/scale>. Acesso em: 21 abr. 2020.

SCALE. In: Online Etymology Dictionary. Disponível em: 〈https://www.etymonline.com/search?q=scale>. Acesso em: 21 abr. 2020.

SHEPPARD, E.; MCMASTER, R. B. (ed.). Scale and geographic inquiry: nature, society, and method. Oxford: Blackwell Publishing Ltd, 2004. 272 p.

SILVA, F. G. D. A escala cartográfica na ponta dos dedos: contribuição das maquetes táteis na construção da noção de proporção no espaço vivido. Dissertação (Mestrado em Geografia). 2015 - Universidade Federal de Goiás, Instituto de Estudos Socioambientais (IESA), Programa de Pós-Graduação em Geografia, Goiânia, 2015. 144 f.: il.

SILVEIRA, M. L. Escala geográfica: da ação ao império? Terra Livre, ano 20, v.2, n. 23. jul./dez., 2004.

SMITH, N. Contours of a spatialized politics: homeless vehicles and the production of geographical scale. Social Text, v. 33, p. 55-81, 1992.

SMITH, N. Geografia, diferencia y políticas de escala. Trad. María Franco García. Terra Livre, ano 18, v. 2, n. 19, p. 127-146, 2002.

SOUZA, M. L. Escala geográfica, "construção social da escala" e "política de escalas". In: SOUZA, M. L. Os conceitos fundamentais da pesquisa sócio-espacial. Rio de Janeiro: Bertrand Brasil, 2013. Cap. 8, p. 179-216.

TAYLOR, P. J. The paradox of geographical scale in marx's politics. Antipode, v. 19, n. 3, p. 287-360, 1987.

VAN WEIJEN, D. The Language of (Future) Scientific Communication. Research Trends, v. 31, dez. 2012. Disponível em: <https://www.researchtrends.com/issue-31-november2012/the-language-of-future-scientific-communication/>. Acesso em: 25 jul. 2020. 
VIEIRA, D. J. Estado Nacional e Escalas Geográficas: uma abordagem exploratória a partir de Neil Smith. Revista Paranaense de Desenvolvimento, Curitiba, n. 123, p. 161-178, jul./dez. 2012. Disponível em:

<http://www.ipardes.gov.br/ojs/index.php/revistaparanaense/article/view/506/760 >. Acesso em: 25 jul. 2020.

ZHANG, C.; ZHU, T; HU, S. Basic Theory of Map Scale. Geospatial Information, v. 7 n. 1, s/p., 2009.

ZHAO, J.; SIMPSON, M.; WALLGRÜN, J. O.; SAJJADI, P.; KLIPPEL, A. et al. Exploring the Effects of Geographic Scale on Spatial Learning. Cognitive Research: principles and Implications, v. 5, n. 14, 2020. DOI: https://doi.org/10.1186/s41235-020-00214-9

Ivanilton José de Oliveira

Professor Associado no Instituto de Estudos Socioambientais (IESA) da Universidade Federal de Goiás (UFG). Graduado em Administração de Empresas pela Faculdade Anhanguera de Ciências Humanas e em Geografia (licenciatura e bacharelado) pela Universidade Federal de Goiás. Mestre e Doutor em Geografia Humana pela Universidade de São Paulo (USP) e com pós-doutorado pela Universidade de Santiago de Compostela (USC), Espanha.

Endereço institucional: Rua Jacarandá s/n, Câmpus Samambaia - Prédio do IESA, sala B-27. CEP 74690-900 Goiânia - Goiás - Brasil.

\section{Patrícia de Araújo Romão}

Professora Associada no Instituto de Estudos Socioambientais (IESA) da Universidade Federal de Goiás (UFG). Graduada em Geologia pela Universidade de Brasília (UnB). Mestre e Doutora em Geotecnia também pela UnB.

Endereço institucional: Rua Jacarandá s/n, Câmpus Samambaia - Prédio do IESA, sala B-27. CEP 74690-900 Goiânia - Goiás - Brasil. 